Background Numerous prospective studies have demonstrated that HIV transmission is greatly reduced in heterosexual HIV serodiscordant couples when the HIV-positive partner (HPP) is receiving combination anti-retroviral therapy (cART) with undetectable viral load (UVL). Comparable data in homosexual male serodiscordant couples (HM-SDC) are extremely limited. We report a pre-specified interim analysis of the relationship between UVL and HIV transmission in the Opposites Attract observational cohort study of HM-SDC in Australia, Bangkok and Rio de Janeiro.

Methods HM-SDC reporting regular anal intercourse with each other were recruited through clinical sites. Detailed information on sexual risk behaviours was collected at each visit from the HIV-negative partner (HNP). HNPs were tested at baseline and follow-up for HIV antibodies and STIs (sexually transmitted infections), and HPPs for HIV viral load and STIs. Incidence rates were calculated per couple-year of follow-up (CYFU) using person-year methods, and stratified by whether different forms of condomless anal intercourse (CLAI) were reported. UVL was defined as $<200$ copies $/ \mathrm{mL}$. One-sided confidence intervals (CI) were calculated using the exact Poisson method. Linked HIV transmission in couples was defined by phylogenetic analysis.

Results By December 2014, 234 HM-SDC were enrolled: 135 from Australia, 52 from Bangkok and 47 from Rio de Janeiro. There were a total of 150.0 CYFU in 152 couples with at least one follow-up visit of whom $65(42.8 \%)$ were in a non-monogamous relationship. At baseline, $84.2 \%$ of HPPs were on cART and in total $82.9 \%$ had UVL. STI prevalence was $11.2 \%$ in HPPs and 6.6\% in HNPs. There were 90.8 CYFU in periods where CLAI was reported with a total of 5,905 acts of CLAI in 88 couples. There were no linked HIV transmissions. The upper limit of the 95\% CI of the transmission rate was 4.06/100 CYFU for periods in which CLAI was reported, and 6.46/100 CYFU for periods in which receptive CLAI was reported.

Conclusions There were no linked HIV transmissions in 150 CYFU in these HM-SDC, despite close to 6,000 acts of CLAI. The upper confidence limit of the transmission rate during follow-up in periods during which CLAI was occurring was 4.06/ 100 CYFU. These data add to emerging evidence that the rate of HIV transmission in HM-SDC is very low when the HIV-positive partner is on ART. Further follow-up of a larger sample size is required to accurately delineate any residual risk.

\section{O20.4 TRANSMISSION RISK BELIEFS INFLUENCE SEXUAL RISK BEHAVIOUR OF HIV-POSITIVE MSM}

${ }^{1} \mathrm{HM}$ Truong*, ${ }^{1} \mathrm{R}$ Fatch, ${ }^{2} \mathrm{~B}$ Nguyen, ${ }^{2} \mathrm{M}$ Grasso, ${ }^{2} \mathrm{~T}$ Robertson, ${ }^{2} \mathrm{HF}$ Raymond, ${ }^{1,2} \mathrm{~W}$ McFarland. 'University of California, San Francisco; ${ }^{2}$ San Francisco Department of Public Health

\subsection{6/sextrans-2015-052270.190}

Introduction Beliefs about the efficacy of antiretroviral treatment for decreasing risk of HIV transmission may influence a person's sexual risk behaviour. We examined whether individuals' HIV transmission risk beliefs predicted subsequent engagement in unprotected anal intercourse (UAI) among men who have sex with men (MSM).

Methods HIV-positive and HIV-negative MSM were recruited for the longitudinal study in San Francisco using time-location sampling. Participants who completed both the baseline and 6-month follow-up behavioural surveys were included in the analysis $(\mathrm{N}=773)$. Beliefs regarding HIV transmission risk at baseline and reported UAI with any sexual partner during the 6month interval between the baseline and follow-up surveys were evaluated.

Results UAI at baseline was associated with an increased likelihood of UAI at follow-up among both HIV-positive MSM (OR $=6.45, \mathrm{p}<0.01)$ and HIV-negative MSM (OR $=13.59, \mathrm{p}<$ $0.01)$. UAI was more frequently reported at follow-up among HIV-positive MSM who agreed with the statements, "Because of combination drug treatment for HIV, I am less concerned about infecting someone," $(\mathrm{OR}=2.49, \mathrm{p}=0.04)$ and "I am less worried about having UAI now that treatments can be taken after unprotected sex," (OR $=6.52, \mathrm{p}=0.02)$. HIV-positive MSM who agreed with statement, "My sexual practices are safer because someone who is positive can become re-infected with HIV," were less likely to report UAI at follow-up $(\mathrm{OR}=0.13$, $\mathrm{p}$ $<0.01)$. Transmission risk beliefs were not associated with UAI at follow-up among HIV-negative MSM.

Conclusion HIV-positive MSM who believed there was less risk of transmitting HIV due to the availability of antiretroviral treatment and post-exposure prophylaxis were more likely to engage in UAI. Concerns about the possibility of re-infection may have influenced some HIV-positive men to refrain from engaging in UAI. These findings suggest the need for prevention messages to highlight treatment adherence and viral suppression as important factors that affect the efficacy of antiretrovirals for reducing HIV transmission risk.

Disclosure of interest statement This work was supported by the US National Institutes of Health [R01 MH077509].

\subsection{TRENDS IN UNDIAGNOSED HIV AND HIV TESTING BEHAVIOUR IN COMMUNITY SAMPLES OF MEN WHO HAVE SEX WITH MEN IN LONDON, UK: RESULTS FROM REPEAT CROSS-SECTIONAL SURVEYS BETWEEN 2000-2013}

${ }^{1}$ Sonali Wayal*, 'V Parsons, ${ }^{1}$ A Copas, 2M Danielle, 3A Nardone, 3A Aghaizu, $1 \mathrm{G}$ Hart, 1R Gilson, 'AM Johnson. 'Centre for Sexual Health \& HIV Research, Research Department of Infection \& Population Health, University College London; ${ }^{2}$ Central and North West NHS Foundation Trust, London, UK; ${ }^{3}$ Public Health England, Colindale, UK

\subsection{6/sextrans-2015-052270.191}

Background HIV testing can reduce undiagnosed and late HIV diagnosis. We examine trends between 2000-2013 of overall and undiagnosed HIV prevalence, HIV testing among men who have sex with men (MSM), and factors associated with undiagnosed HIV.

Methods Repeat cross-sectional anonymous behavioural surveys with oral specimens for HIV antibody (Ab) testing were conducted in community venues in London. Participants were treated as undiagnosed HIV + if they tested HIV Ab+, and had never tested for HIV, last tested or perceived themselves as HIV negative, or did not know their HIV status. Undiagnosed fraction is the proportion of undiagnosed HIV $\mathrm{Ab}+$ results of the total number of HIV Ab+ results. Trends between 2000-2013 and factors associated with undiagnosed HIV (2011 and 2013 data) were examined using logistic regression. Adjusted odds ratios (AOR) and 95\% confidence intervals (CI) were calculated. 
Results The majority of the 11876 participants included in the analysis were White, employed, and median age was 33 years. In 2013, overall and undiagnosed HIV prevalence was 13.6\% (106/ $782)$ and $3.2 \%(25 / 782)$ respectively. Overall undiagnosed fraction remained unchanged: $34 \%(45 / 131)$ in 2000 and $24 \%(25 /$ $106)$ in 2013. Undiagnosed fraction among sexual health clinic non-attenders in last year remained unchanged: 62\% (23/37) in $2000 ; 59 \%(10 / 17)$ in 2013 . HIV testing in the last year increased: $26 \%(263 / 997)$ to $60 \%(467 / 777)$; among undiagnosed HIV+ men, it increased from $28.6 \%$ (10/35) to $66.7 \%$ $(16 / 24)$. Compared to men aged $>45$, men aged 15-25 (AOR: 7.47, 95\% CI: 1.56-35.74); compared to sexual health clinic attenders in the last year, non-sexual health clinic attenders (AOR: 4.39, 95\% CI: 1.90-10.16) were more likely to have undiagnosed HIV.

Conclusions HIV testing has increased yet undiagnosed HIV remains unchanged. Strategies to increase HIV testing among young MSM and in non-sexual health clinics should be developed and evaluated.

Declaration of interest statement AMJ has been a Governor of the Wellcome Trust since 2011. The other authors declare that they have no conflicts of interest.

\subsection{FIRST FORCED SEX AND SEXUAL BEHAVIOUR AFFECTING PREVALENCE OF HIVIAIDS AMONG MSM IN SOUTH INDIA}

Rakesh Kumar Singh*. Ph.D Research Scholar, International Institute for Population Sciences, Mumbai, India

\subsection{6/sextrans-2015-052270.192}

Background Forced sex is the risk factor for psychological morbidities, HIV, and other sexually transmitted infections. Limited study on MSM are available in India and no systematic attempt has been made to know the impact of first forced sex with male and HIV. Therefore, the present study examined the prevalence of the first forced sex and its linkage with HIV infection in South India.

Methods The present study has been used data from the cross sectional survey known as Integrated Behavioural and Biological Assessment during 2009-10. The survey was conducted in the selected districts of states, Andhra Pradesh, Tamil Nadu and Maharashtra. The sample size of MSM was 3875. Bivariate and multivariate logistic regression analysis were used.

Results Those MSM who have reported of their first forced sex with males, are found more likely to be HIV positive $34.69 \%$ vs. $29.06 \%$ and $\mathrm{OR}=1.297, \mathrm{p}<0.05)$ as compared to those MSM who did not have first forced sex with male. In Tamil Nadu, prevalence of first forced sex with male among MSM highest in Salem (57.1\%) followed by Madurai (56.4\%), and Dharmapuri (51.2\%). In Andhra Pradesh, 23.5\% MSM have had first forced sex with male in Hyderabad followed by Guntur (16.8\%), East Godavari (8.8\%) and Vizag (4.0\%).

Conclusion The present study has found that first forced sexual intercourse with a male is a significant risk factor for the HIV infection among MSM in South India. Therefore, there is an urgent need to control the prevalence of first forced sex and transmission of HIV infection.

Disclosure of interest N/A.

\section{1 - HIV and co-morbidity}

\subsection{PLATELET DERIVED SOLUBLE GLYCOPROTEIN VI DECREASES PRIOR TO CORONARY EVENT IN HIV POSITIVE PATIENTS}

${ }^{1,2} \mathrm{JM}$ Trevillyan*, ${ }^{3} \mathrm{EE}$ Gardiner, ${ }^{3}$ RK Andrews, ${ }^{4} \mathrm{~A}$ Maisa, ${ }^{2,4} \mathrm{AC}$ Hearps, $1,2,4 \mathrm{SM}$ Crowe, ${ }^{1,2} \mathrm{JF}$ Hoy. ${ }^{1}$ Infectious Diseases Unit, Alfred Hospital, Melbourne Australia; ${ }^{2}$ Department of Infectious Diseases, Faculty of Medicine, Nursing and Health Science, Monash University, Australia; ${ }^{3}$ Australian Centre for Bloods Diseases, Monash University, Melbourne Australia; ${ }^{4}$ Centre for Biomedical Research, Burnet Institute, Melbourne, Australia

\subsection{6/sextrans-2015-052270.193}

Introduction Platelets play a key role in coronary artery disease (CAD). Glycoprotein VI (GPVI) is a platelet specific collagen receptor which is shed when activated. Soluble (s) GPVI is associated with $\mathrm{CAD}$ in the general population and lower levels have been found in patients taking abacavir. This trial was performed to determine if sGPVI was predictive of CAD in HIV.

Methods $24 \mathrm{HIV}+$ subjects with CAD (HIV+ cases) with stored plasma available in the 12 months before CAD diagnosis were age and sex matched 1:2 with 46 HIV+ subjects without CAD (HIV+ controls). 41 HIV negative controls (healthy controls) were used as comparators. HIV+ patients had two samples analysed; 12 and 1 month before CAD diagnosis; healthy controls had a single sample analysed (202 samples in total). sGPVI was determined by ELISA.

Results Of the combined HIV+ subjects 63 [90\%] were male; mean 51 years; $92.8 \%$ taking antiretrovirals. HIV+ subjects (combined HIV + cases and HIV + controls) were more likely to smoke $(34$ [30.6\%] v's 3 [7.3\%], p < 0.001) than healthy controls. HIV+ cases were hypertensive (13 [54.1\%] v's 5 [10.8\%], $\mathrm{p}<0.001)$ and had a family history of CAD (12 [52.1\%] v's 9 [25.0\%], p 0.033) at higher rates than HIV + controls. sGPVI was higher in HIV+ subjects (combined) then healthy controls (129.9 ng/ml [SD 59.5] v's $84.4 \mathrm{ng} / \mathrm{ml}$ [SD 46.1], $\mathrm{p}<0.001)$. 12 months before event there was no difference in sGPVI between HIV+ cases and HIV+ controls $(123.2 \mathrm{ng} / \mathrm{ml}$ [SD 61.7] v's $137.8 \mathrm{ng} / \mathrm{ml}$ [SD 63.5], p 0.369). 1 month before event sGPVI was significantly lower in HIV + cases $(111.1 \mathrm{ng} / \mathrm{ml}$ [SD 45.0] v's $143.9 \mathrm{ng} / \mathrm{ml}$ [SD 56.1], p 0.016).

Conclusion HIV+ subjects have higher sGPVI than healthy controls; sGPVI is lower prior to CAD event in HIV+. sGPVI may play an important role in promoting CAD in HIV.

Disclosure of interest statement No commercial funding was involved in this project.

\subsection{ONE PROFILE OR MANY? PLASMA BIOMARKERS CXCL10, SCD163 AND SCD14 REVEAL DISTINCT ASSOCIATIONS WITH HIV TREATMENT RESPONSE, CHOICE OF TREATMENT REGIMEN, AND CARDIOVASCULAR RISK FACTORS}

\begin{abstract}
${ }^{1,2} \mathrm{~A}$ Castley, ${ }^{3}$ I James, ${ }^{1} \mathrm{~L}$ Williams, ${ }^{2} \mathrm{C}$ Berry, ${ }^{1,3} \mathrm{D}$ Nolan*. ${ }^{1}$ Department of Clinical Immunology, 2nd Floor, North Block, Royal Perth Hospital, Wellington Street, Perth, Western Australia 6000; ${ }^{2}$ School of Veterinary and Life Sciences, Murdoch University, Perth, Western Australia 6150; ${ }^{3}$ Centre for Clinical Immunology and Biomedical Statistics (CCIBS), Murdoch University, Perth, Western Australia 6150
\end{abstract}

10.1136/sextrans-2015-052270.194 